\title{
Mise bas et non défécation. Nouvelle interprétation de trois propulseurs magdaléniens sur des bases zoologiques, éthologiques et symboliques.
}

\author{
H. G. BANDI *
}

II ne fait pas de doute que les données zoologiques associées aux observations éthologiques aident à mieux comprendre l'art des chasseurs préhistoriques. Mais je pense de plus en plus que l'étude des symboles est, elle aussi, d'une certaine importance dans l'interprétation de l'art rupestre et surtout de l'art mobilier.

Prenons par exemple le cas de trois propulseurs typiques de l'art mobilier magdalénien. Chacun de nous se souvient des célèbres représentations de jeunes ruminants, chamois ou bouquetins du Mas d'Azil, de Bédeilhac et d'Arudy.

PROPULSEUR DU MAS D'AZIL (fig. $1 \mathrm{a}$ et $\mathrm{b}$ )

En 1940, Marthe et Saint Juste Péquart découvrent au Mas d'Azil (Ariège) un propulseur tout à fait exceptionnel, tant sur le plan technique que sur le plan artistique. La pièce, en bois de renne sculpté mesure $29,5 \mathrm{~cm}$ de longueur et figure de nos jours encore, parmi les plus repré-

* Université de Berne. 


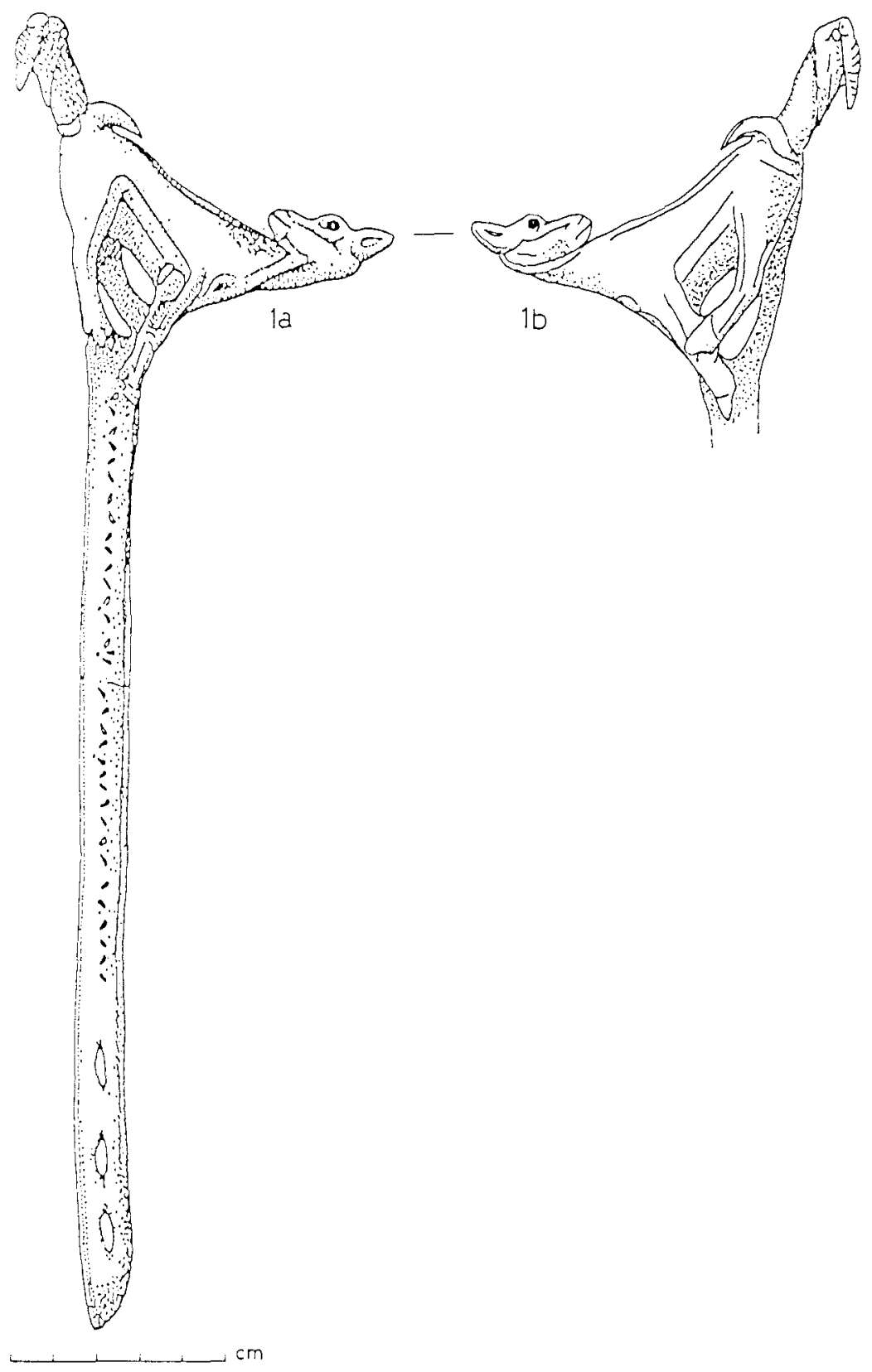

Fig. 1a et b. Propulseur du Mas d'Azil (Ariége). Musée du Mas d'Azil. Dessins de M. F. Le Brun-Ricalens et de Mme E. Bürki. 
sentatives oeuvres de l'art magdalénien. Le manche du propulseur muni à une extrémité de trois trous losangiques, porte sur toute sa longueur de petites incisions arrangées par paires. A l'extrémité opposée se trouve la représentation d'un animal sculpté en ronde bosse. Celui-ci a souvent été interprété comme bouquetin, quelque fois comme chamois. Par contre, la possibilité qu'il s'agisse d'une chevrette (toujours sans andouiller) peut être exclus, cet animal ne jouant aucun rôle au pléistocène tardif.

L'animal représenté n'a pas de cornes, attribut si caractéristique pourtant des bouquetins et des chamois, mâles et femelles. Le profil du front avec una convexité de l'os frontale me semble rappeler plutôt un chamois. Les grandes orbites étaient probablement remplies d'une masse colorée à son état original. Ces caractéristiques morphologiques sont, non seulement bien observées, mais conviennent tout à fait au crâne d'un chamois, comme au volume de la tête de l'animal vivant.

Ces traits semblent souligner le caractère juvénile (faon) de la représentation, mais il ne faut pas oublier que ces grands yeux ronds, un peu saillants se retrouvent aussi sur la tête des adultes. II ne faut non plus lier l'absence des cornes à une éventuelle immaturité de l'animal représenté. Rappelons à ce propos que le développement des cornes du chamois (et du bouquetin) s'effectue lors de la première année (chez un chamois la longueur moyenne est de $4 \mathrm{~cm}$, et parfois atteint, $5,8 \mathrm{~cm}$ ). On peut donc déduire de l'absence de ces cornes une intention soulignée de l'artiste.

D'autant plus que d'autres détails anatomiques sont bien attestés: oreilles, museau, narines, queue, sabots. II ne fait certes pas de doute que si les cornes avaient été sculptées -donc saillantes- elles auraient été très fragiles. La position de la tête de l'animal, choisie intentionellement par la sculpteur —nous en parleront plus tard- l'empêchait de représenter les cornes en relief sur le cou ainsi que cela peut s'observer par exemple sur la sculpture des rennes de Bruniquel (Tarn et Garonne).

Sur le propulseur du Mas d'Azil le chamois dressé tourne la tête vers la droite et regarde en arrière. Sur le coté droit ses deux pattes sont posées au sol, c'est-à-dire sur le monche di propulseur mais si la patte avant sur le côté gauche s'appuie sur le sol, le sabot de la patte arrière en revanche, est posé sur l'articulation du tarse de la patte avant. Sur le ventre de l'animal on remarque une ligne gravée qui suggèrerait une différence de couleur du pelage, dans cette région basse de l'abdomen. La queue courte dessine une boucle dirigée vers la tête, devenant parallèle à la ligne dorsale. 
Mais ce qui a toujours intrigué les préhistoriens se trouve précisement localisé sous la queue. Là, en effet on distingue une masse cylindrique, issue de l'arrière train de l'animal. Cette masse a été nommée "boudin». Sur elle on remarque un annexe qui a été interprété comme correspondant à deux oiseaux se becquetant (fig. 1a). II n'y a peu de doute qu'il y a effectivement un oiseau dont la queue sert du crochet au propulseur. Mais je ne suis pas persuadé que nous ayons à faire à deux oiseaux, le deuxième se posant avec son dos vers le "boudin". Anatomiquement il n'aurait pas eu la possibilité de s'accrocher à ce dernier. La configuration qu'on a voulu interpréter comme un deuxième oiseau purrait donc faire partie du «boudin» (fig. 1b). La présence d'un seul oiseau correspond d'ailleurs aux deux autres propulseurs en question. Notons enfin qu'auprès de la tête de l'oiseau, formant avec sa queue le crochet du propulseur, se trouve un petit trou (ce qui a peut-être contribué à l'hypothèse qu'on ait à faire à deux oiseaux) et que le fanon de l'animal porte une concavité sur le côté droit.

\section{PROPULSEUR DE BÉDEILHAC (fig. 2)}

II s'agit d'un fragment en bois de renne découvert par Romain Robert, à $30 \mathrm{~km}$ de distance à Bédeilhac (Ariège) dix ans après celui du Mas d'Azil et qui lui ressemble par maints détails. Ce propulseur n'a presque plus de manche. Le fragment mesure $9 \mathrm{~cm}$, il présente un animal sculpté en ronde bosse qu'on a désigné comme étant un bouquetin. Pour ma part, je pense qu'il s'agit plutôt d'un chamois en raison de la morphologie générale de la tête. Cette tête porte deux globes oculaires de grande taille qui, ainsi que Romain Robert le rapporte "sont figurés par une masse d'apparence résineuse, à surface légèrement convexe".

On voit, également sortir de l'arrière-train de l'animal, une sorte de masse oblongue ou "boudin". II est de même pourvu d'une figurine qui peut être interprétée comme oiseau dont la queue sert de crochet au propulseur. Le chamois paraît être un jeune animal. La queue prolonge la ligne du dos. Au dessus de la queue, un trou plus ou moins rhombique traverse la sculpture de part en part.

La différence la plus notable entre les deux oeuvres porte sur la position même des animaux: le chamois de Bédeilhac est accroupi, ses pattes sont toutes repliées sous l'abdomen, les sabots se touchent. Mais ces sabots, caractérisés par une concavité, auraient "été primitivement remplis comme les cavités orbitaires d'une substance résineuse noirâtre... 


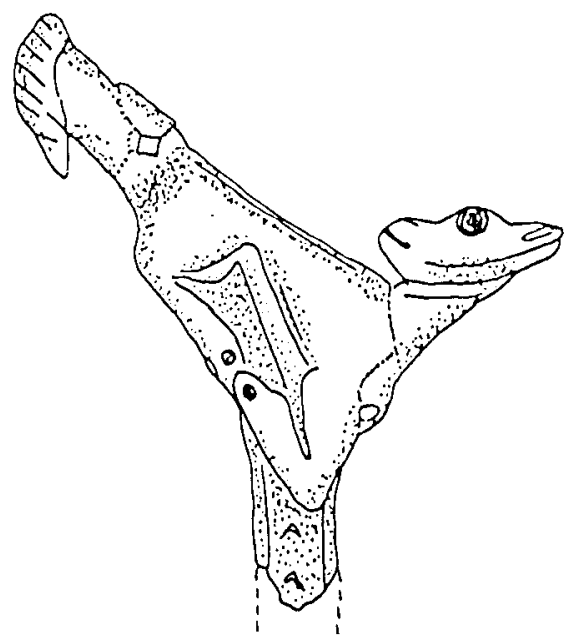

Fig. 2. Propulseur de Bédeilhac (Ariège). Collection Romain Robert. Dessin de Mme. E. Bürki.

Une trace de cette matière subsiste au fond d'une des cupules" précise R. Robert.

Le ventre de ce chamois présente également des lignes gravés qui suggèrent une différence de teinte du pelage vers le bas ventre. Le fanon, bien développé, est percé d'un trou. Le museau avec bouche et narines ainsi que les oreilles, sont executés avec beaucoup de fidélité anatomique. Les cornes sont cependant absentes, sans doute pour des raisons identiques à celles évoquées ci-dessus. Mentionnons encore la présence de quelques incisions d'apparence géométrique sur la partie restante du manche.

Pour toutes ces ressemblances anatomiques et stylistiques entre ces deux propulseurs, il semble qu'on puisse les considérer comme contemporaines de l'apogée de l'art mobilier magdalénien. On a posé la question si les deux sculptures ne pouvaient pas être l'oeuvre du même chasseurartiste; ce n'est pas exclu en vue de la petite distance entre les deux sites et en considérant la datation conforme.

Quoi qu'il en soit, il apparait comme certain que la répétition de ce thème implique un patrimoine symbolique bien établi, une tradition iconographique bien attestée, enfin peut-être une référence à une mythologie ayant un rayonnement au moins régional. 


\section{PROPULSEUR D'ARUDY (fig. 3)}

Le troisième propulseur provient de la Grotte Saint-Michel d'Arudy dans les Pyrenées-Atlantiques (et non de la Grotte d'Espalunge avoisinante). II s'agit d'une pièce fragmentaire en bois de renne, au manche brisé. Sculpté en ronde bosse, l'animal représenté a la tête endommagée. La perte de cette région rend évidemment délicate l'identification de l'animal, cependant il semble que le corps correspond morphologiquement à celui des chamois décrits ci-dessus. Ce corps mesure $10 \mathrm{~cm}$; l'animal se tient dressé sur ses pattes comme dans le cas du Mas d'Azil, cependant les pattes sont ici réunies et rejoignent le bout du manche du propulseur brisé au delà des sabots.

La queue prolonge naturellement la ligne dorsale incurvée, comme sur le chamois de Bédeilhac, elle se courbe légèrement avant de rejoin-

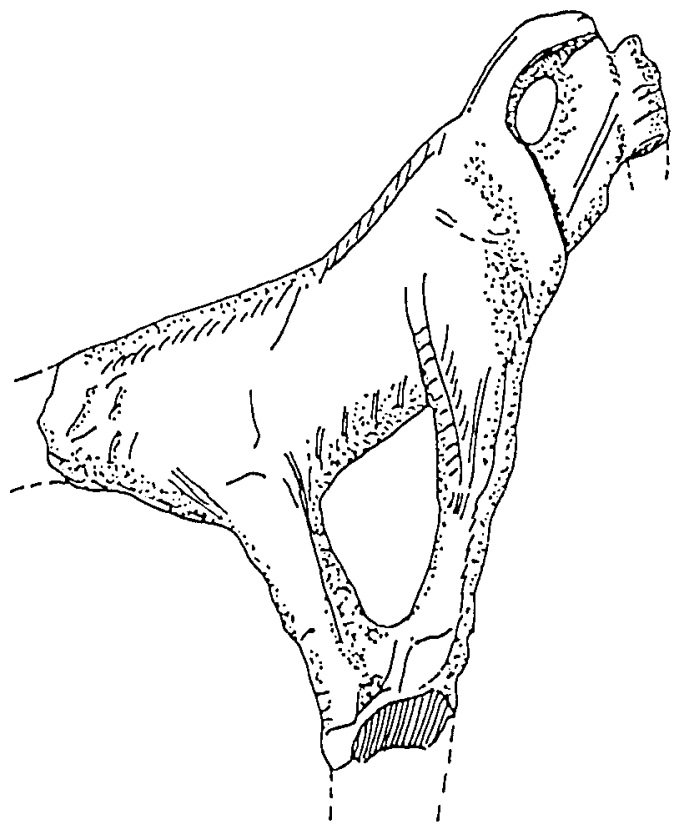

Fig. 3. Propulseur de la Grotte de Saint-Michel d'Arudy (Pyrénées-Atlantiques). Musée des Antiquités Nationales Saint-Germain en Laye (collection Mascaraux). Dessin de Mme. E. Bürki. 
dre la masse-«boudin» énigmatique qu'expulse l'arrière-train de l'animal. On distingue encore un assez grand trou ovale localisé entre la queue et la masse «boudin", et, sur le boudin proprement-dit une figurine brisée rappelant les oiseaux que nous avons signalées pour le Mas d'Azil et Bédeilhac.

Mais ce qui rend la sculpture d'Arudy captivante et exceptionelle réside dans l'identification de la région anatomique qui expulse la masse“boudin" jusqu'alors indéchiffrable. En effet, contrairement à toutes les hypothèses anciennement formulées, il ne s'agit pas de l'anus de l'animal mais de sa vulve. Cette identification est tout à fait bien attestee, elle est capitale pour le renouvèlement de l'interprétation du thème choisi, puis sculpté, par les chasseurs magdaléniens.

\section{NOUVELLE INTERPRÉTATION: MISE BAS ET NON DÉFÉCATION}

Rappelons que l'interprétation la plus communément exprimée à propos de ces trois propulseurs, met en évidence un thème et retient une intention: seaux»

- le thème est celui du «faon à l'oiseau». Ou du «faon aux oi-

- l'intention est changée d'humour et de plaisanterie puisqu'il s'agit de l'évocation d'un animal en position de défécation, la tête tourné vers l'arrière-train, au moment précis où un ou deux oiseaux viennent se poser, s'amuser, sur les excréments sortant de l'anus (voir le dessin et commentaire dans le livret de Laurent, Heureuse Préhistoire).

R. Robert faisait déjà remarquer que "dans la réalité, les "fumées" des Antilopidés, des Capridés et des Cervidés sont sub-sphériques, petites, nombreuses et séparées les unes des autres". Comme cela ne correspond pas à ce qui a été sculpté, il pense qu'il y aurait une erreur d'observation commise par l'artiste magdalénien.

G. Camps, plus récement suppose pour des raisons pratiques, une modification morphologique intentionelle de la matière fécale. Celle-ci est représentée compacte, dense, grande. Quant à la représentation des oiseaux, elle aurait été ajoutée pour permettre de créer un crochet pour le propulseur.

Les constatations faites par Romain Robert concernant les fumées des animaux en question correspondent aux données naturelles. On ne peut pas accepter la supposition que le chasseur-artiste magdalénien qui 
connaissait son gibier dans tous les détails, ait pu commetre une telle erreur. A part de cela il faut revoir l'hypothèse que le «boudin" sorte de l'anus. Dans ce cas on devrait admettre que l'anus des animaux du Mas d'Azil et de Bédeilhac était très vaste. Pour le chamois d'Arudy, il me semble évident qu'il s'agit de la vulve.

Un autre point concerne la tête de l'animal, tournée en arrière chez les exemples du Mas d'Azil et de Bédeilhac. En observant le comportement des animaux on peut facilement remarquer qu'on ne voit pratiquement jamais un animal regardant en arrière pendant la défécation. Par contre on trouve cette position souvent chez les femelles mettant bas des jeunes. II faut donc penser que le motif en discussion représente une scène de mise bas. La position des trois animaux correspond parfaitement à cette explication. Les chamois (comme les bouquetins ou les chevreuils) mettent bas leurs jeunes en position debout ou couchés par terre. L'animal du propulseur d'Arudy se tient debout, les quatre pattes posées sur le sol; de même le chamois du Mas d'Azil est représenté debout sauf qu'une de ses pattes derrières est levée et se pose vers la jambe de devant. Le chamois de Bédeilhac est couché par terre.

Le "boudin" sortant de la vulve des trois animaux peut être interprété comme sac embryonnaire. L'apport d'informations éthologiques et comportementales est capital dans ce domaine. Pour appuyer cette nouvelle interprétation j'ai eu recours aux avis de divers spécialistes, notamment à ceux de mon collègue Hannes Sägesser, professeur de zoologie, directeur du jardin zoologique de Berne, qui a eu l'aimabilité de mettre à ma disposition des photographies prises lors de la mise bas d'une chevrette en captivité.

La première photo (fig. 4) montre la chevrette debout après la mise bas d'un premier chevreau. Celui-ci s'est déjà dégagé du sac embryonnaire, on peut voir pendre quelques fragments de ce sac. La chevrette tourne la tête vers son arrière-train. Sur la deuxième photo (fig. 5), l'animal est allongé, il expulse un second sac embryonnaire de sa vulve, tandis que le premier chevreau cherche déjà à têter. La mère regarde vers la droite. Sur la troisième photo (fig. 6), on assiste à l'expulsion du sac contenant le deuxième chevreau, la chevrette continue de regarder vers la droite, le cou raidi, jusqu'à ce que la totalité du sac soit rejetée hors de son corps.

Sur la quatrième photo (fig. 7), la chevrette regarde de nouveau en direction de son arrière-train, où le sac embryonnaire s'est détaché complètement de la vulve, sans que le deuxième chevreau s'en soit déjà libéré. 


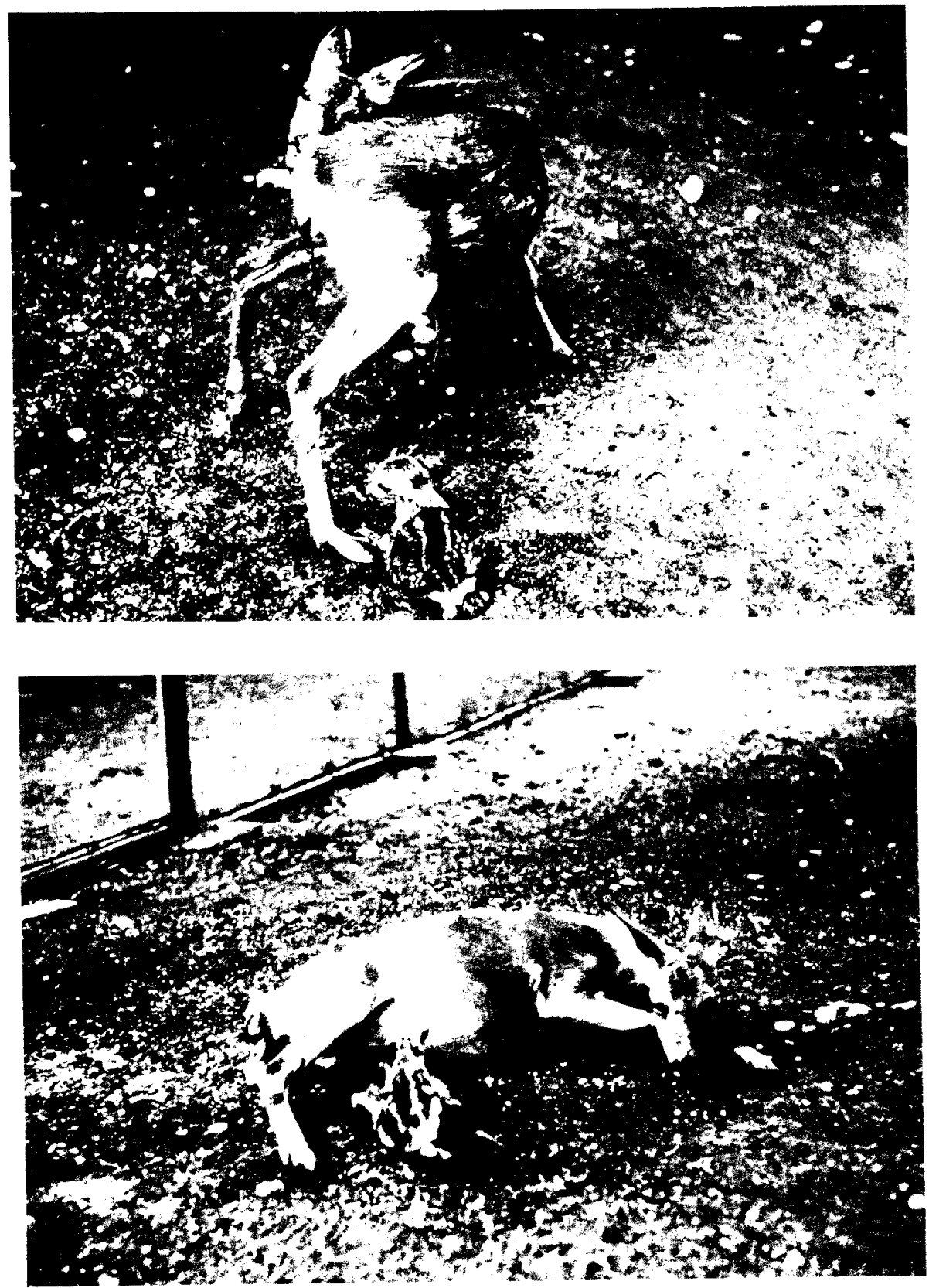

Figs. 4 et 5. Mise bas d'un chevereuil au "Tierpark" de Berne. Photos du Prof. H. Sägemer. 

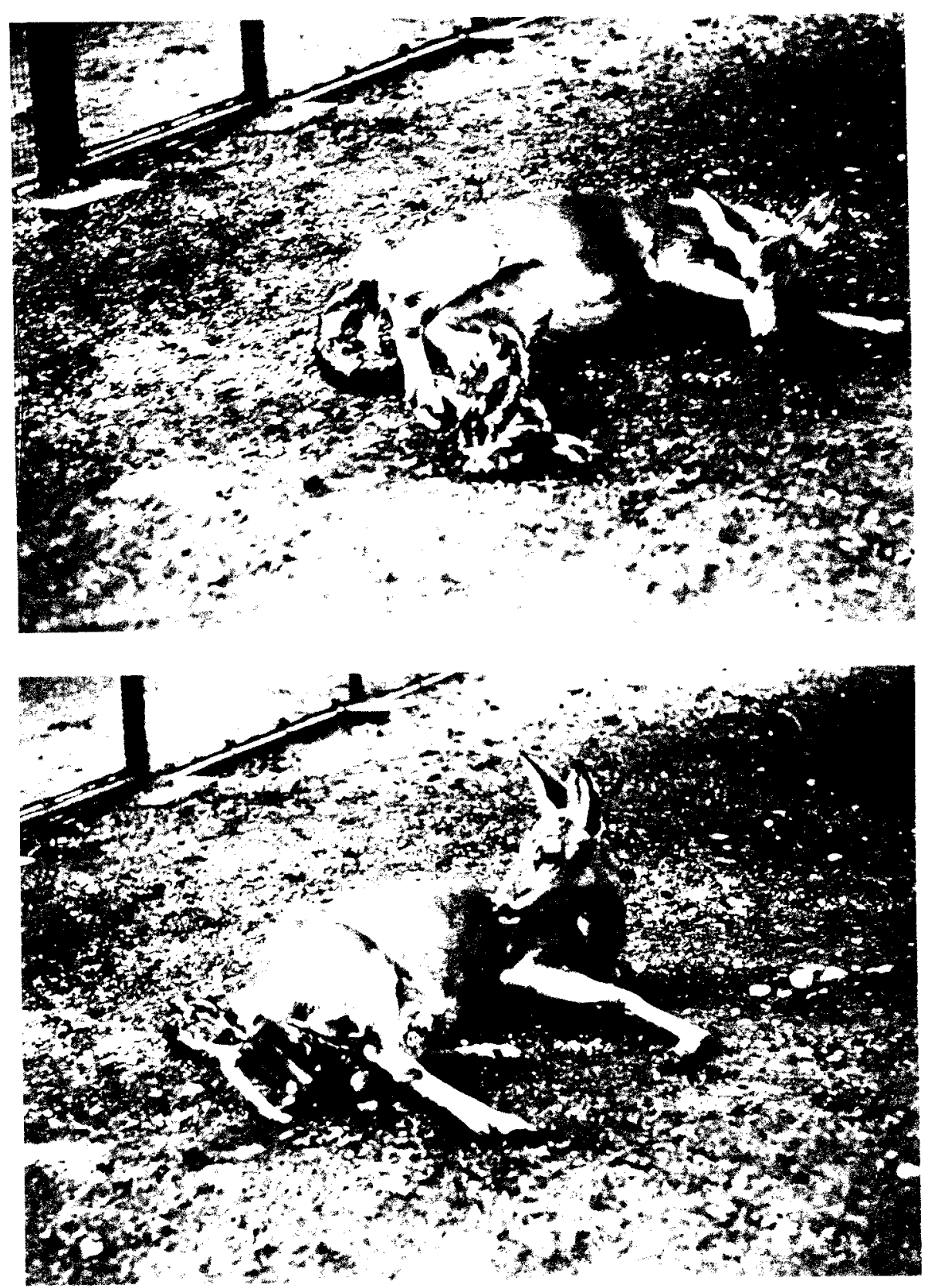

Figs. 6 et 7. Mise bas diun chevreuil au "Tierpark" de Berne. Photos du Prof. $H$. Sägesser. 
Selon $\mathrm{H}$. Sägesser, la mise bas des chamois et des bouquetins s'effectue selon le même processus.

Résumons doc sur les trois propulseurs quels sont les détails anatomiques qui ne conviennent pas vraiment à l'hypothèse d'une défécation et trahiraient, en revanche, une réelle méconnaissance comportementale, chez les chasseurs magdaléniens:

1. Anus - boudin: si cette région de l'arrière-train correspondait à l'anus, on serait en droit d'être surpris par la taille exagérément grande donnée à la masse fécale - dite boudin —, et à la forme de celle-ci lors du rejet.

2. Tête tournée vers l'arrière: tous les spécialistes s'accordent à dire que la défécation chez l'animal, en général, (sauf cas de maladie, etc.) ne s'accompagne jamais d'un regard. Ce rejet est bref et sans intérêt particulier.

Quels sont maintenant les détails qui attestent la mise bas, la naissance.

1. Boudin: ce qui a été nommé «masse-boudin» et qui sort de la vulve de la jeune bête d'Arudy est en réalité le sac embryonnaire. Mon collègue $H$. Sägesser confirme sans réticence cette interprétation. Le fait que le sac embryonnaire est expulsé pratiquement vers le haut et qu'il est tout droit doit être expliqué avec la nécessité du sculpteur de former le crochet du propulseur.

2. Age des animaux représentés: comme il a été montré, la tête ne doit pas être celle d'un faon mais correspond aussi bien à un jeune adulte; le corps a une taille normale et peut très bien être interprété comme appartenant à une jeune femelle. Les chamois d'aujourd'hui peuvent se reproduire dès la deuxième année bien que normalement la première mise bas n'a lieu qu'a l'âge de quatre ans. Remarquons finalement qu'aucune des trois sculptures montrant beaucoup de détails, porte le moindre indice d'un organe sexuel.

D'autres questions restent encore soulevées par ces trois propulseurs magdaléniens:

1. Les oiseaux: s'agit-il vraimant d'oiseaux, comme le suggère le propulseur du Mas d'Azil? Cela est probable. Sur ce document particulièrement scigné, il semble possible de reconnaitre l'oeil et le bec de l'oiseau sculpté. Les figurines correspondantes des propulseurs de Bédeilhac et d'Arudy sont executées moins soigneusement (en plus, celle 
d'Arudy est brisée). L'analogie nous permet la même interprétation. Detail intéréssant: l'oiseau du Mas d'Azil aussi bien que les deux autres de Bédeilhac et d'Arudy portent sur les ailes des traits transversaux gravés et de signification incertaine. Las ressemblances de ces trois propulseurs, au niveau de ce détail stylistique sont tout à fait étonnantes. Ceci a contribué à émettre l'hypothèse d'un même artiste ayant executé ces oeuvres.

Le rôle joué par les oiseaux reste, lui aussi, encore indéfini. N'a-t-on pas été jusqu'à supposer que l'artiste y avait eu recours, comme à un artifice, pour créer le crochet? Cette hypothèse est peu convaincante. Ne faudrait-il pas prudemment souligner notre ignorance ou bien recourir à une explication symbolique? - Rappelons que l'imaginaire préhistorique renferme de nombreuses inconnues et qu'il n'est pas impossible que le thème oiseau-destin, ou oiseau-âme, soit à évoquer, à propos de ces documents. Ne recourt-on pas - de nos jours encore - au moment de la naissance d'un bébé à l'évocation d'une cigogne dans certaines régions? Les petits oiseaux peuvent figurer - p.e. chez les peuples ouralo-altaiques d'Asie centrale- les âmes des enfants. Même si le thème de l'oiseau est rarament représenté dans l'art pléistocène, nous connaissons quelques cas qui attestent leur rôle symbolique: dans la caverne des Trois Frères en Ariège, on observe trois impressionants hibous (deux adultes et un jeune); à Lascaux, en Dordogne, la célèbre scène du puits présente: un bison blessé (?), un sagaie et un propulseur (?), un homme ityphallique ayant une tête d'oiseau, une perche sur laquelle se tient un oiseau... L'interprétation soulève encore de nombreuses discussions; pourtant il semblerait que nous soyions en face d'un rite d'initiation figurant les symboles de la mort et de la renaissance. Même le shamanisme n'est pas exclu et on sait qu'il peut connaître la conception d'âmes-oiseaux. L'âge attribué à cette scène se situe dans la même phase du Magdalénien que les trois propulseurs.

En somme: loin d'opter pour une solution hypothétique, il nous parait préférable d'envisager une interprétation symbolique dans laquelle le jeune chamois-femelle, l'oiseau, la renaissance et peut-être la mort (par l'intermédiaire de l'arme qui tue), se trouveraient unis pour évoquer un mythe, qui nous échappe. Le support de cette "histoire» serait donné par un propulseur: objet maniable, présentable, chargé d'un sens symbolique largement reconnu par une communauté de chasseurs magdaléniens.

2. Fonction du propulseur: S'agit-il d'un instrument fonctionnel? R. Robert niait déjà le caractère quotidien et fonctionnel du document du Mas d'Azil dont la dimension est de $29,5 \mathrm{~cm}$. Quant aux deux autres 
propulseurs, de taille plus petite, il paraît également improbable qu'ils aient pu être utilisés pour la chasse. Si l'on se refère aux instruments esquimaux équivalents comme ceux décrits et publiés par E. W. Nelson pour l'Alaska, on constate que ces propulseurs récents mesurent au minimum $40 \mathrm{~cm}$. Toutefois des expériences actuelles montrent l'efficacité de propulseurs très courts. Et il ne faut non plus oublier que rien ne s'oppose à ce que des "manches" prolongent en bois les propulseurs magdaléniens. En outre il n'est pas exclu que les Magdaléniens connaissaient des "flèches à lancer», c'est à dire des sagaies qui avaient les dimensions d'une flèche mais qu'on lançait à l'aide d'un propulseur; en tout cas quelques pointes d'arme en bois de renne du Magdalénien sont si petites qu'elles correspondent mal à un sagaie ordinaire. Si on admet que nos propulseurs - sans prolongation en bois - auraient servi à une fonction cérémoniale, on peut même s'imaginer qu'ils étaient utilisés pour lancer de telles "flèches" à l'occasion de certains rites. II est peu probable qu'il s'agisse de jouets aidant à former les futures chasseurs, comme chez les Inuits ou, dans des sites préhistoriques, l'on trouve souvent des armes, des outils, des lampes, etc., en miniature. R. Robert ne croit pas à un usage pratique mais il pense au contraire à des pièces emblématiques et non fonctionelles: "la gracilité de l'ensemble, manche compris, semble devoir exclure toute idée d'une utilisation effective, comme le prouverait encore l'angle formé par le crochet et la hampe".

Une utilisation cérémoniale ne s'oppose pas à l'hypothèse d'un caractère symbolique des sculptures au bout des trois propulseurs. Les travaux d'Alexander Marshack laissent même penser que les incisions au manche pourraient avoir une signification symbolique.

Cette étude met l'accent sur le caractère non figé de nos hypothèses, sur le renouvèlement de notre approche de l'art mobilier du Paléolithique supérieur. J'ai souhaité montrer en utilisant cette méthode "des petits pas" que les données réunies de la zoologie, de l'éthologie autour d'une pensée symbolique -même complexe et imparfaitement déchiffrableouvrent encore de nouvelles perspectives à nos recherches. Ces trois propulseurs magdaléniens qui nous content chacun la même histoire d'une naissance d'un jeune faon, délivrent un message artistique et symbolique d'une très grande force avec sobriété et concision.

Mes remerciements vont au Professeur $H$. Sägesser avec qui j'ai pu discuter le problème d'une mise bas chez les ruminants et qui m'a procuré les photographies montrant la naissance de chevraux au "Tierpark" de Berne; à Mme G. Haas, Berne, à l'aide de laquelle je me suis familia- 
risé avec l'importance des symboles; à Mme E. Bürki, Berne, qui était la première à me parler de son expérience avec la mise bas chez des moutons et qui m'a rendu service en dessinant les fig. 1b, 2 et 3; à Mme C. Roubet de l'I.P.H. à Paris, qui s'est chargée de revoir le manuscript aussi bien en ce qui concerne son contenu scientifique que du point de vue stilistique. 


\section{BIBLIOGRAPHIE}

"L'Art des Cavernes", 1984: Atlas des grottes ornées paléolithiques françaises. Paris.

Bandi, H. G., Huber, W., Sauter, M. R., et. Sitter, B., (ed.), 1984: La contribution de la zoologie et de l'éthologie à l'interprétation de l'art des peuples chasseurs préhistoriques. Fribourg.

Baumann, F., 1949: Die freilebenden Säugetiere der Schweiz. Bern.

BreuIL, H., 1952: Quatre cents siècles d'art parietal. Montignac.

CAMPS, G., 1984: “La défécation dans l'art paléolithique». In: BANDI, $H$. G., Huber, W., Sauter, M. R., et. Sitter, B. (ed.), La contribution de la zoologie et de l'éthologie à l'interprétation de l'art des peuples chasseurs préhistoriques. Fribourg.

ClotTES, J. (ed.), 1987: Pré-Actes, Colloque int d'art mobilier paléolithique, Foix-Mas d'Azil 16.-21.11.1987. Toulouse.

Graziosı, P., 1956: L'arte dell'antica età della pietra. Firenze.

LAURENT, P., 1965: Heureuse Préhistoire. Perigueux.

Lerol-Gourhan, A., 1965: Préhistoire de l'art occidentale. Paris.

LURKER, M., 1979: Wörterbuch der Symbolik. Stuttgart.

MARSHAK, A., 1972: The roots of civilisation. New York.

Nelson, E. W., 1986/87: "The Eskimo about Bering Strait". Bureau of American Ethnology, 18th report. Washington.

PÉquart, M. et S. J., 1942: "Récente découverte de deux oeuvres d'art magdalénien au Mas d'Azil». Revue Scientifique, $\mathrm{n}^{\circ}$ 3205: 91 ss.

Robert, R., 1953: “Le Faon à l'Oiseau. Tête de propulseur sculpté du Magdalénien de Bedeilhac». Préhistoire Spéléologie Ariègeoises, VIII: $11-18$. 
